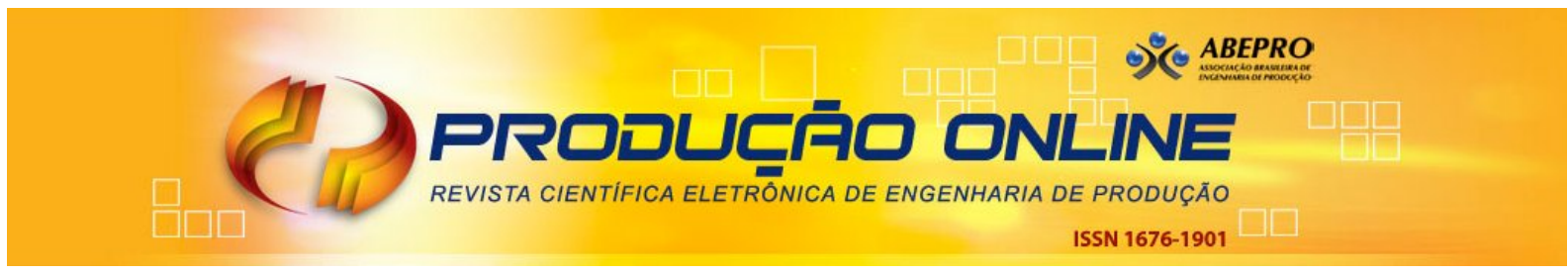

\title{
A PRODUÇÃO ACADÊMICA INTERNACIONAL EM GESTÃO DE OPERAÇÕES: UM ESTUDO BIBLIOMÉTRICO
}

\section{INTERNATIONAL ACADEMIC PRODUCTION IN OPERATIONS MANAGEMENT: A BIBLIOMETRIC STUDY}

\author{
Valdir Antonio Vitorino Filho* E-mail: valdir.vitorino@gmail.com \\ Silvio Roberto Ignácio Pires* E-mail: sripires@unimep.br \\ Mário Sacomano Neto** E-mail: msacomano@ufscar.br \\ Eliciane Maria da Silva ${ }^{\star \star \star}$ E-mail: elicianems@gmail.com \\ João Batista de Camargo Júnior* E-mail: ibcir@unimep.br \\ Jane Corrêa Alves Mendonça* E-mail: janeagronegocio@hotmail.com \\ *Universidade Metodista de Piracicaba (UNIMEP), Piracicaba, SP. \\ **Universidade Federal de São Carlos (UFSCAR), São Carlos, SP. \\ ***Fundação Getúlio Vargas (FGV), São Paulo, SP.
}

Resumo: Este artigo tem como objetivo analisar a produção acadêmica sobre o tema Gestão de Operações, em artigos publicados em periódicos internacionais indexados no ISI Web of Knowledge. A pesquisa avaliou publicações realizadas no período de 2002 a 2012, utilizam-se técnicas bibliométricas para averiguar questões como: quais são os autores que possuem mais publicações no tema; quais foram os anos, países e periódicos em que esses artigos foram publicados e ainda, a apresentação dos autores mais co-citados nas referências. No total a pesquisa envolveu 110 artigos e 4545 referências com o termo Operations Management; 274 artigos e 6644 referências com o termo Production Management; e 49 artigos e 1250 referências utilizados na pesquisa para o termo Manufacturing Management. Os resultados da pesquisa mostram que (a) não existe uma concentração de autores com vários artigos publicados sobre o tema; (b) verifica-se que os anos com maior número de publicações são os de 2007 e 2010 somando $24,48 \%$ do total analisado; (c) os Estados Unidos é o país que concentra o maior número de publicações com $26,33 \%$ do total de artigos analisados; (d) foram identificados Williamson (1975), Dyer e Singh (1998), Granovetter (1985) e Uzzi (1997) como os autores mais co-citados nas referências bibliográficas.

Palavras-chave: Gestão de operações. Gestão da produção. Gestão da manufatura. Bibliometria. citações.

Abstract: This article aims to examine the academic production about Operations Management in articles published in international journals indexed in ISI Web of Knowledge. The research evaluates publications from 2002 to 2012 and uses bibliometric techniques to investigate questions like: what are the authors who have most publications on the subject, what are the years, countries and journals in which the articles were published and, also, the presentation of the most co-cited authors in the references. In total the study verified 110 articles and 4545 references to the term Operations Management, 6644 articles and 274 references to the term Production Management and 49 articles and 1250 references to the term Manufacturing Management. The research results show that (a) there isn't a concentration of authors who have published several articles about the subjects; (b) the years with the largest number of publications are 2007 and 2010, totaling 24,48 \% of the total analyzed; (c) the United States is the country which has the greatest number of publications, with $26,33 \%$ of all 
analyzed articles; (d) Williamson (1975), Dyer and Singh (1998), Granovetter (1985) and Uzzi (1997) were identified as the most co-cited authors in the references.

Keywords: Operations management. Production management. Manufacturing management.bibliometric. References.

\section{INTRODUÇÃO}

De acordo com Slack e Lewis (2002), durante as últimas duas décadas as estratégias de produção evoluíram para estratégias de operações, onde faz sugir uma nova abordagem para operações com a inclusão de todos os tipos de operações, e não apenas as que fabricam produtos físicos. É importante diferenciar claramente as operações em produtos e serviços. Mas em ambos é necessário explicitar o plano de como os recursos dentro do processo serão utilizados, controlar as atividades no dia-a-dia e ainda, pensar em questões de melhoria para o desempenho do processo.

Diante desse contexto esta pesquisa tem como objetivo analisar a produção acadêmica na área de Gestão de Operações - Operations Management, em artigos publicados em periódicos internacionais indexados no ISI (Web of Knowledge). Com a aplicação de métodos bibliométricos, essa pesquisa tem caráter quantitativo, exploratório e descritivo. Leva em consideração também a análise dos termos Gestão da Produção - Production Management e Gestão da Manufatura Manufacturing Management, uma vez que não se identifica na literatura uma anuência entre pesquisadores sobre os limites de cada definição. Enquanto para alguns autores (HALL, 1988; MOREIRA, 2008) a gestão de operações está ligada somente a transformação de mão de obra em serviços, outros pesquisadores (SLACK; LEWIS, 2002; FRANCISCHINI, 2004) defendem que o termo engloba o planejamento de todas as funções que levam a produção de um bem ou serviço, incluindo-se desenvolvimento e distribuição. Do mesmo modo, enquanto Hall (1988) defende que o conceito de gestão de produção se refere ao gerenciamento da transformação de matéria prima em produto final, para Pires (2009) o termo já engloba em seu âmago a transformação de entradas, quer sejam elas matérias primas ou mão de obra, em saídas, representadas por produtos acabados ou 
serviços prestados. Por esse motivo, no escopo desse trabalho os três termos são considerado relevantes para a condução da pesquisa e alcance de seus objetivos.

Parte-se de questões ligadas às referências bibliográficas utilizadas nos estudos em Gestão de Operações, tendo como questionamento principal: "quais são as referências mais utilizadas nos estudos com o tema Gestão de Operações? E se existe alguma predominância nas referências bibliográficas utilizadas nos artigos sobre Gestão de Operações?".

Com a elaboração do objetivo e questões de pesquisa deste estudo, delimitam-se as seguintes preposições: (a) há concentração de publicações sobre Gestão de Operações em poucos periódicos; (b) existe predominância de publicações em alguns países; (c) há claramente alguns autores com maior número de publicações na área; e (d) as publicações na área cresceram conforme o passar dos anos.

Através da pesquisa com método bibliométrico busca-se identificar o material publicado sobre Gestão de Operações e possibilitar uma visão a nível internacional dos pesquisadores que escrevem sobre o tema e a identificação dos principais autores co-citados nesses artigos.

Para Araújo (2010) a chamada lei de Lotka parte do princípio de que uma grande quantidade de produção científica está concentrada em trabalhos de poucos autores. E na chamada lei de Brandford se forem enumerados periódicos em ordem decrescente, levando em consideração o critério de produtividade de artigos sobre um determinado tema, pode-se obter o que é chamado de núcleo de periódicos mais particularmente destinados ao tema escolhido. Análises essas que serão detalhadas na seção categorias para análise dos dados.

Diversas pesquisas fazem uso do método bibliométrico, para diversas áreas no contexto da Gestão de Operações, como por exemplo, inovação e tomada de decisão no processo de logística reversa (TENÓRIO; SILVA; DACORSO, 2014); gestão da inovação e avaliação de desempenho (DIENSTMANN et al., 2014); redes sociais e cooperação (LOPES; CARVALHO; FLEURY, 2013); estratégias de manufatura (KNOFF et al., 2014); e sustentabilidade e vantagem competitiva (GABRIELE et al., 2012). 
Podem-se destacar algumas pesquisas no campo da Gestão de Operações, que fazem uso de uma análise da rede, a nível Brasil, destacam-se Martins, Rossoni, Csillag, Martins e Pereira (2010) que se baseiam em 2.668 artigos publicados entre 1997 e 2009, apresentam como resultados da pesquisa a formação de grupos coesos e próximos que promovem uma estabilidade ao campo, apesar da verificação de uma rede fragmentada e pouco densa. Rungtusanhatham, Choi e Hollingworth (2003) apresentam um estudo sobre os temas abordados em 285 artigos, distribuídos em seis revistas sobre Gestão de Operações, em um período de 1980 a 2000, sendo que o estudo identificou os temas que se destacaram como mais utilizados: a gestão de tecnologia, estratégias de operações, gestão da qualidade, gestão da cadeia de suprimentos e Just in Time de Manufatura.

\section{GESTÃO DE OPERAÇÕES}

Para elucidar o contexto da pesquisa, a seguir são debatidos os conceitos de gestão de manufatura, gestão ou administração da produção e gestão de operações. Embora ainda não exista um consenso entre pesquisadores sobre definições únicas para os termos, faz-se importante verificar as abordagens relevantes sobre esses temas.

Hall (1988) menciona que o termo manufatura se relaciona a todas as atividades operacionais que participam do processo produtivo de uma empresa. Nesse âmbito estão incluídas as atividades de projeto do produto, pesquisa e desenvolvimento, marketing, distribuição, vendas, finanças e contabilidade. Consequentemente, a gestão da manufatura pode ser conceituada como a tarefa de planejar globalmente a produção dentro das empresas, que buscam transformar seus processos em propulsores de vantagem competitiva ao adquirirem e manterem competências em realizar determinadas atividades melhores do que seus concorrentes (HILL, 1993). Desse modo, para Skinner (1969) a gestão da manufatura é, muitas vezes, vista pelas organizações como forma de medir o desempenho empresarial, seu aumento de eficiência e a redução de custos. Tal autor ainda acrescenta que a gestão de manufatura deveria ser considerada pelas 
empresas como uma função para gerar competitividade, visando contribuir com o alcance dos objetivos corporativos e com o consequente sucesso organizacional.

Do mesmo modo, Hall (1988) expõe que, diferentemente da manufatura, a produção se preocupa especificamente com a conversão da matéria prima em produto final, envolvendo as atividades de fabricação, montagem, teste, controle de qualidade, administração de materiais, gestão de pessoas, planejamento de padrões, programação de produção, entre outras. Assim, a gestão da produção trataria da transformação de materiais em produto final através da execução de atividades interligadas com a utilização dos recursos disponíveis, em um horizonte de tempo já determinado (WHEELWRIGHT; HAYES, 1985).

Para Moreira (2008) o termo produção está mais ligado às atividades industriais de fabricação de produtos, enquanto a palavra operações refere-se às atividades produtivas realizadas em empresas de serviços. O que ocorre, porém, é que muitas empresas não trabalham somente fabricando produtos ou fornecendo serviços. Especialmente na atualidade, muitas organizações oferecem a seus clientes produtos e serviços, agregados ou não, no intuito de se diferenciar da concorrência e alcançarem uma parcela maior do mercado.

Por esse motivo, observa-se que a literatura da área utiliza também a denominação gestão de produção e operações ao se referir as atividades de transformação de entradas, sejam elas matérias primas ou mão de obra, em saídas, representadas por produtos acabados ou serviços prestados. Nesse sentido, em uma definição mais holística, Pires (2009) menciona que a gestão de produção e operações pode ser entendida como a gestão de um conjunto de atividades e/ou processos que transformam entradas (matéria-prima, mão de obra) em saídas (produtos acabados, serviços prestados) com maior valor agregado do que as entradas.

Por outro lado, Fusco e Sacomano (2007) nos mostram que as áreas produtivas de uma organização são organizadas por várias subunidades que podem, por sua vez, serem consideradas versões reduzidas da operação global de que fazem parte. Assim, a operação global pode ser considerada uma macrooperação enquanto os departamentos são as microoperações. A implicação do entendimento 
desses autores é que se podem considerar todas as partes da empresa como operações a requerer administração da produção.

Cada organização possui um tipo de operação, uma vez que cada organização produz um tipo diferente de produto ou serviço, levando, assim, ao conceito de gestão de operações como uma função que faz parte da organização e que visa gerenciar todos os processos que auxiliem na produção de produtos e serviços (SLACK; LEWIS, 2002). Nessa mesma linha de pensamento, Francischini (2004) cita que a atividade que envolve todas as ações referentes ao fluxo de matérias e/ou serviços em uma organização, desde o fornecimento físico, planejamento, execução, controle e sua distribuição para clientes, é denominada gestão de operações. Assim, as atividades mencionadas por Hall (1988) como de manufatura são vistas por esses autores como atividades produtivas, dando um significado comum aos termos gestão de operações e gestão de manufatura. Como se pode observar, embora para alguns autores os termos gestão de manufatura, gestão da produção e gestão de operações tragam significados semelhantes, outros pesquisadores identificam diferenças significativas entre eles. Assim, no âmbito desse artigo, não se adotará uma ou outra visão como correta, entendendo-se que os autores dos artigos pesquisados para o estudo bibliométrico utilizam as denominações de acordo com os métodos, objetivos e resultados de suas respectivas pesquisas.

\section{MÉTODO}

A pesquisa realizada caracteriza-se como sendo um estudo bibliométrico, com método quantitativo e de natureza exploratória e descritiva. Foi utilizado como base para coleta dos artigos o ISI Web of Knowledge, sobre o tema: Gestão de Operações, para determinar os autores mais influentes sobre o tema. Segundo Roesh (2001) a pesquisa exploratória fornece flexibilidade em relação ao uso de outros métodos e agrega valor no desenvolvimento de hipóteses. A pesquisa exploratória também envolve a avaliação de teorias e conceitos existentes e sua aplicação aos problemas, quando novas teorias e conceitos podem ser desenvolvidos. E para Collins e Hussey (2005) a pesquisa descritiva é caracterizada 
pelo comportamento dos fenômenos, para obter informações sobre características de um determinado problema.

Para o tratamento dos dados obtidos foram utilizados a ferramenta Microsoft Excel; os softwares Sitkis 2.0 e Ucinet 6.0, para tabulações, formatações e criação das tabelas e figuras para posterior análise, dados esses apresentados na próxima seção.

Cabe também ressaltar que a literatura apresenta alguns estudos bibliométricos, em outras áreas do conhecimento, que tiveram como base métodos de investigação parecidos com o utilizado nessa pesquisa. Pode-se citar como exemplos: Paulista, Campos e Turrioni (2010) com análise da gestão do conhecimento; e Borba, Hoeltgebaum e Silveira (2011) com a investigação científica em empreendedorismo.

\subsection{Amostragem e Coleta de Dados}

Optou-se pela coleta dos dados, na busca de uma representatividade internacional, nos artigos da base ISI Web of Knowledge. As expressões utilizadas para busca na base internacional foram "Operations Management", "Production Management" e "Manufacturing Management". E a coleta de dados ocorreu no mês de abril de 2012, procurado nos títulos, palavras-chave e resumos dos artigos.

Obteve-se para a pesquisa com o termo "Operations Management" um total de 909 artigos, sendo que desses foram selecionados 110 artigos, com uma amostra representando 12, 10\% do total de artigos, onde os mesmos foram selecionados, pelo fato de apresentarem um conteúdo relevante na área para o desenvolvimento da pesquisa. Para o termo "Production Management" um total de 274, sendo selecionada a sua totalidade (100\%). E para o termo "Manufacturing Management" encontrados e selecionados 49 artigos. No total foram analisados 433 artigos. Como demonstrado na Tabela 1: 
Tabela 1 - Amostragem dos Artigos

\begin{tabular}{|c|c|c|}
\hline Tema & Encontrados & Selecionados \\
\hline Operations Management & 909 & 110 \\
\hline Production Management & 274 & 274 \\
\hline Manufacturing Management & 49 & 49 \\
\hline Total & 1232 & 433 \\
\hline
\end{tabular}

Fonte: Elaborado pelos autores com base nos dados da pesquisa.

Para o termo "Operations Management" foram obtidas 4.545 citações de autores, nas referências bibliográficas, com média de 41,32 citações por artigo. Já para a pesquisa com o termo "Production Management" listou-se 6.644 citações de autores nas referências bibliográficas, com média de 24,25 citações por artigo. E para "Manufacturing Management" obtidas 1.250 citações e média de 25,51 citações por artigo. Perfazendo um total de 12.439 citações nas referências bibliográficas dos 433 artigos analisados.

O recorte de tempo para pesquisa abrangeu os anos de 2002 a 2012, compreendendo um período de quase 10 (dez) anos de pesquisa em publicações sobre o tema Gestão de Operações, para a limitação do escopo da análise de pesquisa. A pesquisa utilizou ainda como filtro os documentos do tipo "artigos"; nas áreas de economia de negócios, ciências operacionais de gestão de pesquisa e engenharia; nas categorias gerais de ciência e tecnologia e ciências sociais aplicadas.

\subsection{Categorias para análise dos artigos}

A pesquisa buscou identificar alguns elementos dessas publicações, analisados na seção seguinte, tais como: (a) autores com maior número de artigos publicados; (b) o número de publicações por ano; (c) o número de publicações por países; (d) o número de publicações por periódicos; (e) os autores mais co-citados e as suas relações. 


\section{RESULTADOS DA PESQUISA}

A seguir os resultados obtidos pelo levantamento bibliométrico e uma breve discussão, levando em conta as categorias de análise listadas anteriormente.

$\mathrm{Na}$ busca de um melhor entendimento e separação das informações, os dados seguintes serão divididos por três termos de pesquisa: "Operations Management", "Operations Production" e "Manufacturing Management".

\subsection{Publicações por ano}

$\mathrm{Na}$ Tabela 2, apresenta-se a quantidade de artigos produzidos no período de 2002 a 2012, sendo que os anos com maior número de publicações foram os de 2007 e 2010 com 60 e 56, respectivamente, em sua totalidade. Já o ano de 2011 com 53 publicações. Os de 2009, 2003, 2002 e 2008, respectivamente, com 42, 39, 38 e 38 publicações cada e com os menores índices de publicações no geral os anos de 2006 (26), 2005 (35), 2004 (34) e 2012 (12). Salienta-se aqui que no ano de 2012 a coleta ocorreu no mês de abril, não possibilitando ter um parâmetro do ano por completo.

Tabela 2 - Publicações por ano e tema de pesquisa

\begin{tabular}{|c|c|c|c|c|c|c|c|c|c|c|c|c|}
\hline Tema/Ano & 2002 & 2003 & 2004 & 2005 & 2006 & 2007 & 2008 & 2009 & 2010 & 2011 & 2012 & Total \\
\hline Operations Management & 18 & 8 & 12 & 10 & 4 & 18 & 10 & 6 & 10 & 12 & 2 & 110 \\
\hline $\begin{array}{l}\text { Production Management } \\
\text { Manufacturing }\end{array}$ & 17 & 24 & 18 & 22 & 18 & 36 & 22 & 31 & 40 & 37 & 9 & 274 \\
\hline Management & 3 & 7 & 4 & 3 & 4 & 6 & 6 & 5 & 6 & 4 & 1 & 49 \\
\hline Total & 38 & 39 & 34 & 35 & 26 & 60 & 38 & 42 & 56 & 53 & 12 & 433 \\
\hline
\end{tabular}

Fonte: Elaborado pelos autores com base nos dados da pesquisa

A média de publicações no período (2002 a 2012), para uma amostragem de 433 artigos, foi de 43,3 artigos por ano, sendo a maior concentração das publicações nos anos de 2007 e 2010. Para a publicação com o tema "Operations Management" os anos com maior concentração de artigos publicados foram os de 2002 e 2007, ambos com 18 publicações. Para o tema "Production Management" os anos de 2010 (40) e 2007 (36) artigos publicados. Por sua vez, o termo de pesquisa 
"Manufacturing Management" obteve como maior concentração de publicações o ano de 2003 com apenas 7 publicações. Destaca-se que para todos esses termos de pesquisa utilizados obtiveram-se publicações em cada um dos anos.

\subsection{Publicações por Países}

A seguir são elencados os países com maior número de publicações, para os termos de pesquisa listados. Com a separação dos três termos de pesquisa utilizados (Operations Management, Production Management e Manufacturing Management) e suas representações nos países, suas respectivas quantidades e porcentagens referentes à quantidade total de artigos.

Tabela 3 - Número de publicações por países

\begin{tabular}{|c|c|c|c|c|c|c|c|c|}
\hline Países & $\begin{array}{c}\text { Operations } \\
\text { Management }\end{array}$ & $\%$ & $\begin{array}{c}\text { Production } \\
\text { Management }\end{array}$ & $\%$ & $\begin{array}{l}\text { Manufaturing } \\
\text { Management }\end{array}$ & $\%$ & TOTAL & $\%$ \\
\hline Estados Unidos & 52 & 47,27 & 53 & 19,34 & 9 & 18,37 & 114 & 26,33 \\
\hline Demais Países* & 15 & 13,64 & 32 & 11,68 & 7 & 14,29 & 54 & 12,47 \\
\hline Inglaterra & 12 & 10,91 & 27 & 9,85 & - & - & 39 & 9,01 \\
\hline China & 3 & 2,73 & 30 & 10,95 & 4 & 8,16 & 37 & 8,55 \\
\hline Taiwan & 2 & 1,82 & 21 & 7,66 & 8 & 16,33 & 31 & 7,16 \\
\hline França & 3 & 2,73 & 24 & 8,76 & 2 & 4,08 & 29 & 6,70 \\
\hline Espanha & 3 & 2,73 & 15 & 5,47 & 3 & 6,12 & 21 & 4,85 \\
\hline Alemanha & 2 & 1,82 & 15 & 5,47 & 2 & 4,08 & 19 & 4,39 \\
\hline Japão & - & - & 14 & 5,11 & 4 & 8,16 & 18 & 4,16 \\
\hline Holanda & 4 & 3,64 & - & - & 7 & 14,29 & 11 & 2,54 \\
\hline Itália & 4 & 3,64 & 7 & 2,55 & - & - & 11 & 2,54 \\
\hline Austrália & 2 & 1,82 & 8 & 2,92 & - & - & 10 & 2,31 \\
\hline Brasil & - & - & 6 & 2,19 & 3 & 6,12 & 9 & 2,08 \\
\hline Irã & - & - & 8 & 2,92 & - & - & 8 & 1,85 \\
\hline Israel & - & - & 8 & 2,92 & - & - & 8 & 1,85 \\
\hline Eslovênia & - & - & 6 & 2,19 & - & - & 6 & 1,39 \\
\hline Canadá & 2 & 1,82 & - & - & - & - & 2 & 0,46 \\
\hline Dinamarca & 2 & 1,82 & - & - & - & - & 2 & 0,46 \\
\hline Escócia & 2 & 1,82 & - & - & - & - & 2 & 0,46 \\
\hline Grécia & 2 & 1,82 & - & - & - & - & 2 & 0,46 \\
\hline TOTAL & 110 & 100,00 & 274 & 100,00 & 49 & 100,00 & 433 & 100,00 \\
\hline
\end{tabular}

Países que aparecem apenas uma vez para cada um dos termos de pesquisa utilizados.

Fonte: Elaborado pelos autores com base nos dados da pesquisa. 
Percebe-se a grande concentração de publicações nos Estados Unidos $(47,27 \%)$ com uma representatividade de quase metade da amostra analisada.

Para a pesquisa em Production Management, a maior concentração aparece novamente com os Estados Unidos (53), seguido da China (30), Inglaterra (27), França (24), Taiwan (21), Alemanha e Espanha (15 cada), Japão (14), Austrália, Irã e Israel (com 8 cada), a Itália (7), o Brasil (6), a Eslovênia (6) e demais países com menos de 6 publicações cada representam 32 publicações, ou 11,68\% do total. Destaca-se aqui novamente a predominância dos Estados Unidos no número de publicações. E ainda a boa representatividade dos países asiáticos como China, Taiwan, Japão, Irã e Israel, que juntos somam uma representatividade de 29,52\%.

Para o termo de pesquisa Manufacturing Management, os Estados Unidos ainda aparecem como país com maior número de publicações com 9 artigos, seguidos de Taiwan com 8 artigos, Inglaterra 7 artigos, Japão e China com 4 cada, Brasil e Espanha com 3 artigos cada, França e Alemanha com 2 cada, e demais países com 7 publicações com uma representatividade de 14,29\%.

Nos três termos utilizados para pesquisa os Estados Unidos aparecem como país com maior número de publicações, somando um total de 114 publicações, assim como aparecem nas classificações dos três termos utilizados para pesquisa os países europeus: Inglaterra com 46 artigos no total, França com 29 artigos e Espanha com 21 artigos. E os países asiáticos China com 37 publicações no total, e Taiwan com 31 artigos publicados.

\subsection{Publicações por Periódicos}

$\mathrm{Na}$ Tabela 4 são apresentados os periódicos com maior número de publicações para os termos utilizados na pesquisa. 
Tabela 4 - Número de publicações por periódicos

(continua)

\begin{tabular}{|c|c|c|c|c|c|c|c|c|}
\hline Periódicos & $\begin{array}{c}\text { Operations } \\
\text { Management }\end{array}$ & $\%$ & $\begin{array}{c}\text { Production } \\
\text { Management }\end{array}$ & $\%$ & $\begin{array}{l}\text { Manufaturing } \\
\text { Management }\end{array}$ & $\%$ & TOTAL & $\%$ \\
\hline $\begin{array}{l}\text { Demais Periódicos* } \\
\text { International Journal of } \\
\text { Operations \& } \\
\text { Production }\end{array}$ & 22 & 20,00 & 138 & 50,36 & 20 & 40,82 & 180 & 41,57 \\
\hline $\begin{array}{l}\text { Management } \\
\text { International Journal of }\end{array}$ & 23 & 2,73 & 25 & 12,04 & 2 & 20,41 & 50 & 11,55 \\
\hline $\begin{array}{l}\text { Production Research } \\
\text { International Journal of } \\
\text { Production Economics }\end{array}$ & 8 & 7,27 & 13 & 4,74 & 10 & 8,16 & 25 & 5,77 \\
\hline $\begin{array}{l}\text { Journal of Operations } \\
\text { Management } \\
\text { International Journal of } \\
\text { Advanced } \\
\text { Manufacturing }\end{array}$ & 21 & 19,09 & - & 5,11 & - & 10,20 & 21 & 4,85 \\
\hline $\begin{array}{l}\text { Technology } \\
\text { Production and }\end{array}$ & - & & 14 & & 5 & & 19 & 4,39 \\
\hline Operations & & 10,91 & & - & & - & & \\
\hline $\begin{array}{l}\text { Management } \\
\text { Industrial Management }\end{array}$ & 12 & - & - & 328 & - & - & 12 & 2,77 \\
\hline Data Systems & - & & 9 & & - & & 9 & 2,08 \\
\hline $\begin{array}{l}\text { Production Planning } \\
\text { Control }\end{array}$ & 3 & 2,73 & 6 & 2,19 & - & - & 9 & 2,08 \\
\hline $\begin{array}{l}\text { Journal of Intelligent } \\
\text { Manufacturing }\end{array}$ & - & - & 8 & 2,92 & - & - & 8 & 1,85 \\
\hline $\begin{array}{l}\text { Assembly Automation } \\
\text { International Journal of }\end{array}$ & - & - & 7 & 2,55 & - & - & 7 & 1,62 \\
\hline Computer Integrated & & - & & 1,82 & & 4,08 & & \\
\hline $\begin{array}{l}\text { Manufacturing } \\
\text { IEEE Transactions on }\end{array}$ & - & & 5 & & 2 & & 7 & 1,62 \\
\hline Semiconductor & & - & & 2,19 & & - & & \\
\hline Manufacturing & - & & 6 & & - & & 6 & 1,39 \\
\hline
\end{tabular}

Revista Produção Online, Florianópolis, SC, v.15, n. 1, p. 21-49, jan./mar. 2015. 
Tabela 4 - Número de publicações por periódicos

(conclusão)

\begin{tabular}{|c|c|c|c|c|c|c|c|c|}
\hline Periódicos & $\begin{array}{c}\text { Operations } \\
\text { Management }\end{array}$ & $\%$ & $\begin{array}{c}\text { Production } \\
\text { Management }\end{array}$ & $\%$ & $\begin{array}{l}\text { Manufaturing } \\
\text { Management }\end{array}$ & $\%$ & TOTAL & $\%$ \\
\hline \multicolumn{9}{|l|}{ Omega International } \\
\hline Journal of & & 5,45 & & - & & - & & \\
\hline Management Science & 6 & & - & & - & & 6 & 1,39 \\
\hline $\begin{array}{l}\text { Computers Industrial } \\
\text { Engineering }\end{array}$ & - & - & 5 & 1,82 & - & - & 5 & 1,15 \\
\hline \multicolumn{9}{|l|}{ Journal of Construction } \\
\hline Engineering and & & - & & 1,82 & & - & & \\
\hline Management Asce & - & & 5 & & - & & 5 & 1,15 \\
\hline Interfaces & 3 & 2,73 & - & - & - & - & 3 & 0,69 \\
\hline \multicolumn{9}{|l|}{ M Som Manufacturing } \\
\hline Service Operations & & 2,73 & & - & & - & & \\
\hline Management & 3 & & - & & - & & 3 & 0,69 \\
\hline $\begin{array}{l}\text { Expert Systems with } \\
\text { Applications }\end{array}$ & - & - & - & - & 2 & 4,08 & 2 & 0,46 \\
\hline $\begin{array}{l}\text { International Journal of } \\
\text { Food Engineering }\end{array}$ & 2 & 1,82 & - & - & - & - & 2 & 0,46 \\
\hline $\begin{array}{l}\text { International Journal of } \\
\text { Industrial Engineering }\end{array}$ & - & - & - & - & 2 & 4,08 & 2 & 0,46 \\
\hline $\begin{array}{l}\text { Journal of Engineering } \\
\text { Manufacture }\end{array}$ & 2 & 1,82 & - & - & - & - & 2 & 0,46 \\
\hline $\begin{array}{l}\text { Journal of Food } \\
\text { Engineering }\end{array}$ & 2 & 1,82 & - & - & - & - & 2 & 0,46 \\
\hline Journal of Materials & & & & & & & & \\
\hline Processing & & - & & - & & 4,08 & & \\
\hline Technology & - & & - & & 2 & & 2 & 0,46 \\
\hline Total & 110 & 100,00 & 274 & 100,00 & 49 & 100,00 & 433 & 100,00 \\
\hline
\end{tabular}

Fonte: Elaborado pelos autores com base nos dados da pesquisa.

* Periódicos que aparecem apenas uma vez para cada um dos termos de pesquisa utilizados. 
Para Operations Management, constatam-se que os periódicos com maior número de publicações, sobre o tema de Gestão de Operações, são: International Journal of Operations Production Management (23); Journal of Operations Management (21); e Production and Operations Management (12).

$\mathrm{Na}$ pesquisa em Production Management, tem-se o International Journal of Production Research como o periódico com maior número de publicações, com 33 artigos, seguido do Interntional Journal of Operations \& Production Management (25); o International Journal of Advanced Manufacturing Technology (14); e International Journal of Production Economics (13).

Para o termo de pesquisa Manufacturing Management os artigos foram publicados em: International Journal of Production Research (10), International Journal of Advanced Manufacturing Technology (5), International Journal of Production Economics (4).

Com base no exposto, pode-se dizer que os periódicos com maior número de publicações, no geral, foram: International Journal of Operations \& Production Management (50), International Journal of Production Research (36), International Journal of Production Economics (25), e o Journal of Operations Management (21), com um total de 132 publicações, e uma representatividade de 30,48\% o total da amostra. Demais periódicos aparecem com apenas uma publicação cada.

\subsection{Autores com Maior Número de Artigos Publicados}

Nesta etapa são apresentados os resultados referentes aos autores com maior número de artigos publicados, levando em considerações os termos "Operations Management", "Production Management" e "Manufacturing Management".

Para o termo "Operations Management" foram identificados 248 autores e coautores nos 110 artigos analisados, o que representa uma média de 2,25 autores e co-autores por artigo. Identificou os autores com maior número de publicações de artigos são VOSS, C. (VOSS; TSIKRIKTSIS; FROHLICH, 2002; VOSS, 2007; KARLSSON; VOSS, 2009); e GORMAN, M., F. (GORMAN, 2007; GORMAN, 2011a; GORMAN, 2011b), com 3 (três) publicações cada autor. 
A maioria dos textos não utiliza uma abordagem sobre um tema específico como: Just in Time (JIT), Gestão da Cadeia de Suprimentos (GSCS), Programa Mestre de Produção (PMP), entre outros, abordando questões mais relacionadas a metodologia de pesquisa, remetendo a questões mais gerais.

Para o termo de pesquisa "Production Management" encontrou-se 539 autores e co-autores, em 274 artigos, perfazendo uma média de 1,96 autores e coautores por artigo.

Os autores com maior número de artigos publicados são: Leung, S., C., H. (LEUNG; WU; LAI, 2006; LEUNG; TSANG; NG, 2007; LEUNG; NG, 2007a; LEUNG; NG, 2007b; LEUNG; LAI; NG, 2007), com 5 publicações; seguido de Ng, W., L. (LEUNG; TSANG; NG, 2007; LEUNG; NG, 2007a; LEUNG; NG, 2007b; LEUNG; LAI; NG, 2007), com 4 publicações, e Bivin, D., G. (BIVIN, 2003; BIVON, 2006a; BIVIN, 2006b; BIVIN, 2008), também com 4 publicações $E$ ainda foram encontrados 8 autores com 3 citações cada, e 35 com 2 citações cada. Destaca-se que o autor $\mathrm{Ng}$, W., L., participa de todas as suas quatro publicações com o autor Leung, S., C., H., todas no ano de 2007. E que o periódico que possui maior número é o International Journal of Production Economics, sendo as quatro publicações do autor Bivin, D., G., que por sua vez também possui uma publicação no Economic Inquiry.

Com relação ao termo Production Management, certifica-se que existe uma predominância na produção dos trabalhos, voltada para realização de pesquisas quantitativas, mas especificamente em pesquisa operacional (Operations Research).

E para o termo "Manufacturing Management" foram constatados 119 autores e co-autores, nos 49 artigos analisados, sendo uma média de 2,43 autores e coautores por artigo.

Identificou-se aqui os autores com maior número de publicações de artigos no levantamento realizado, são dois autores com 2 (duas) publicações cada, sendo: Godinho Filho, M. (GODINHO FILHO; FERNANDES, 2009; GODINHO; USZAY, 2011), e Sheu. D., D. (SHEU; PENG, 2003; HUANG; SHEU, 2004). 


\subsection{Autores mais Co-Citados}

A seguir é relatado sobre os autores mais co-citados nos temas de pesquisa Operations Management (17 autores), Production Management (18 autores) e Manufacturing Management (20 autores).

\subsubsection{Operations Management}

$\mathrm{Na}$ Figura 1, são apresentados os autores mais co-citados nas 4.545 referências bibliográficas utilizadas nos 110 artigos estudados. Aqui optou-se por impor um mínimo de 5 (cinco) citações para que o autor pudesse compor essa rede de co-citação.

Dentre esses autores, verifica-se que os autores que possuem maior número de citações com os autores dessa rede de co-citação são respectivamente: Arora e Gambardella (1990) com 23 citações e sendo co-citados com 10 dos autores da rede. Clark e Fujimoto (1991) com 18 citações e co-citados 11 autores. Cohen e Levinthal (1990) com 34 citações e co-citados com 10 autores. Doz (1996) com 29 citações e co-citado com 12 autores. Dyer e Singh (1998) com 60 citações e cocitados 13 autores. Hagedoorn (1993) com 44 citações e co-citado com 12 autores. Henderson e Clark (1990) com 25 citações e co-citados com 12 autores. Kamien e Schwartz (1982) com total de 14 citações e co-citados com 3 autores. Lawrence e Lorsch (1967) com 15 citações e co-citados com 7 autores. Parkhe (1993) com 30 citações e co-citado com 13 autores. Pfeffer e Salancik (1978) com 19 citações e cocitados com 9 autores. Pinto, Pinto e Prescott (1993) com 5 citações e co-citados com 3 autores. Teece; Rumelt e Schendel (1992) com 33 citações e co-citados com 13 autores. Teece (1986) com 26 citações e co-citado com 11 autores. Thompson (1967) possuem 21 citações e co-citados com 12 autores. Já Williamson (1985) com 52 citações e co-citado com 14 autores. E por fim, Williamson (1975) tem 37 citações e aparece co-citado junto com 10 autores. 
Figura 1 - Co-Citação de autores em Operations Management

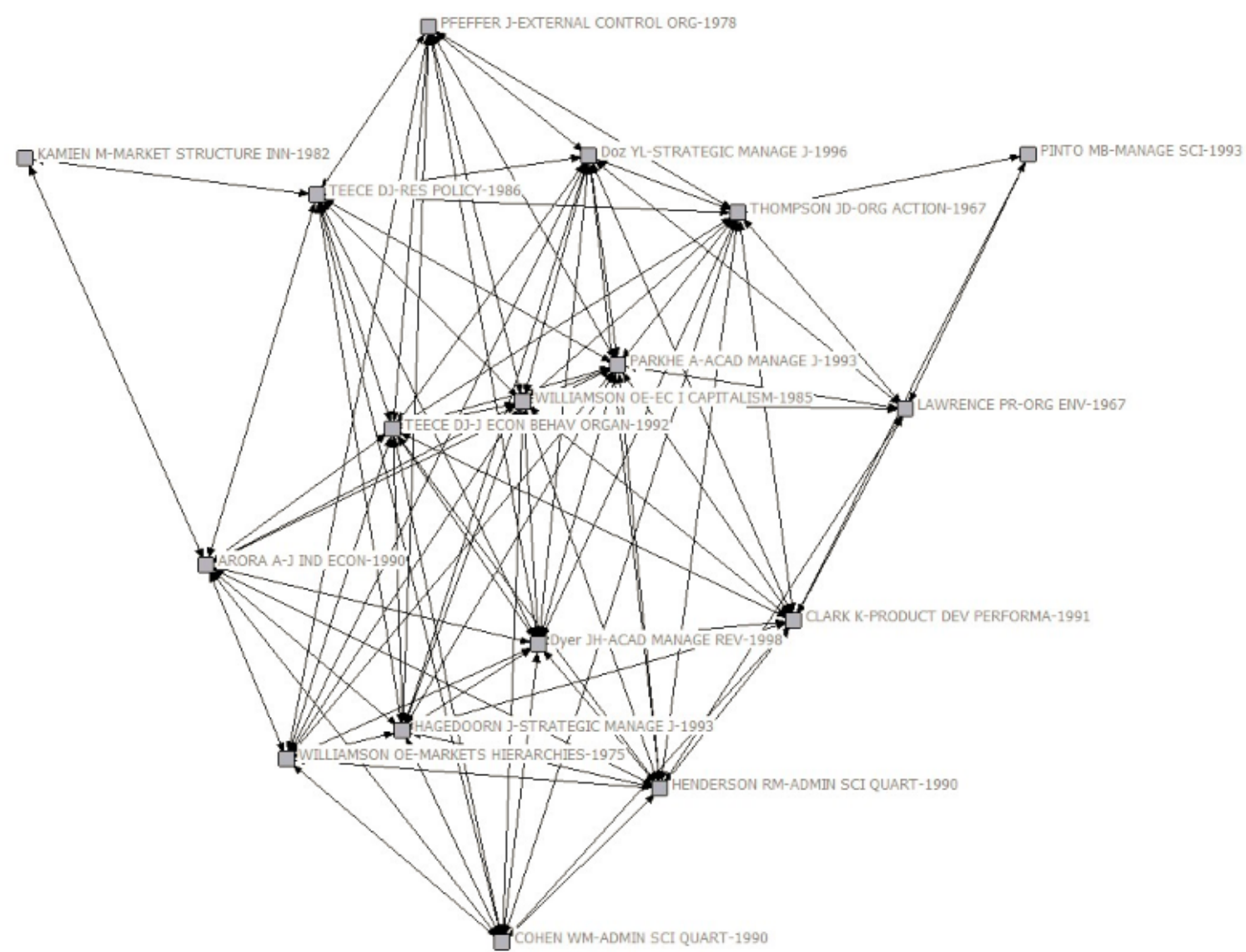

Fonte: Dados da Pesquisa, gerado através do UCINET ${ }^{\circledR}$.

Destaca-se nos resultados que os autores com maior número de citações dentro da rede de co-citação estabelecida com no mínimo de 5 (cinco) citações foram: Dyer e Singh (1998) com 60 citações do trabalho "The relational view: Cooperative strategy and sources of interorganizational competitive advantage", publicado no Academy of Management Review. E Williamson (1985) com 52 citações do texto "The Economic Institutions of Capitalism". Também, Hagedoorn (1993) com 44 citações, do artigo intitulado "Understanding the Rationale of Strategic Technology Partnering since the Early Seventies", publicado no Review of Industrial Organization.

Com algumas áreas, teorias envolvidas e relacionadas como as de estratégias empresariais e de desempenho (ARORA; GAMBARDELLA, 1990; TEECE; SCHENDEL; RUMELT, 1992; THOMPSON, 1967). O desenvolvimento de 
produtos (CLARK; FUJIMOTO, 1991). Questões ligadas à inovação (COHEN, 1990; HENDERSON; CLARKk, 1990; KAMIEN; SCHWARTZ, 1982; TEECE, 1986) e tecnologia de produtos (HAGEDOORN, 1993; HENDERSON; CLARK, 1990). Trabalhos ligados à cooperação empresarial (DYER; SINGH, 1998; PINTO; PRESCOTT, 1993), integração (LAWRENCE, 1967; TEECE, 1986) e alianças estratégicas (DOZ, 1996; PARKHE, 1993). Também a abordagem sobre a ótica da dependência de recursos (PFEFFER; SALANCIK, 1978; WILLIAMSON, 1975; WILLIAMSON, 1985).

\subsubsection{Production Management}

$\mathrm{Na}$ Figura 2, são apresentados os autores mais co-citados nas 6.644 referências bibliográficas utilizadas nos 274 artigos estudados. Sendo apresentados abaixo a rede de co-citação desses autores, ou seja, que autor foi citado juntamente com qual outro autor, optou-se por impor um mínimo de 20 (vinte) citações para que o autor pudesse compor essa rede de co-citação.

Figura 2 - Co-Citação de autores em Production Management

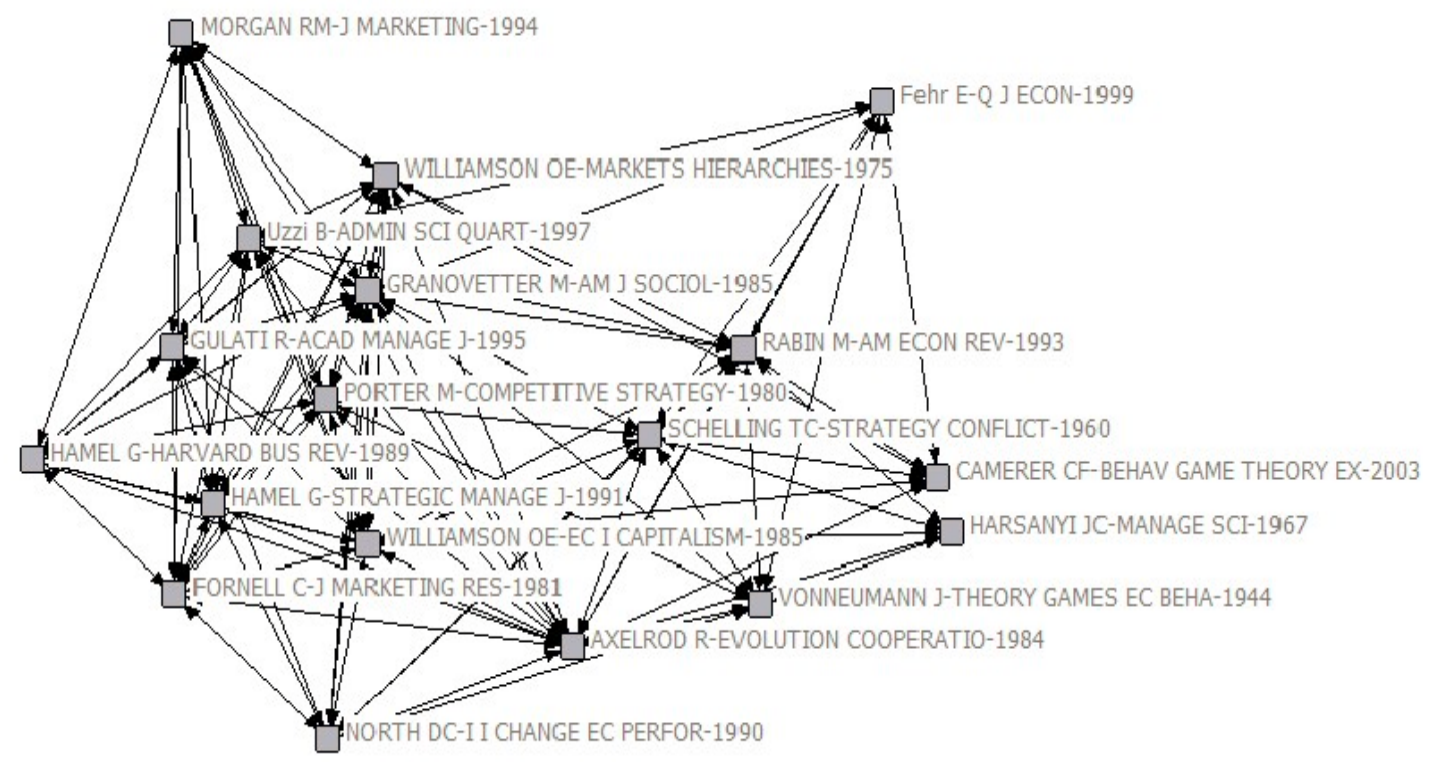

Fonte: Dados da Pesquisa, gerado através do UCINET ${ }^{\circledR}$.

Revista Produção Online, Florianópolis, SC, v.15, n. 1, p. 21-49, jan./mar. 2015. 
No recorte de pesquisa Production Management, destacam-se os temas: estratégia competitiva (PORTER, 1980), redes de empresas, estrutura social e alianças corporativas (GRANOVETTER, 1985; UZZI, 1997; GULATI, 1995), dependência de recursos e economia das instituições (WILLIAMSON, 1985; WILLIAMSON, 1975), a racionalidade limitada na tomada de decisão (AXEROLD, 1984), concorrência, competitividade e teoria dos jogos (HAMEL; PRAHALAD, 1989; HAMEL, 1991; SCHELLING, 1960; HARSANYI, 1967; CAMERER, 2003; NEUMANN; MORGENSTERN, 1944; RABIN, 1993), cooperação e competição (FEHR; SCHIMIDT, 1999), modelos estruturais (FORNELL; LARCKER, 1981), instituições e desempenho econômico (NORTH, 1990), compromisso e confiança nos relacionamentos de marketing (MORGAN; HUNT, 1994).

\subsubsection{Manufacturing Management}

$\mathrm{Na}$ Figura 3, são apresentados os autores mais co-citados nas 1.250 referências bibliográficas utilizadas nos 49 artigos estudados. É apresentada abaixo a rede de co-citação desses autores, ou seja, que autor foi citado juntamente com qual outro autor. Optou-se por impor um mínimo de 20 (vinte) citações para que o autor pudesse compor essa rede de co-citação.

$\mathrm{Na}$ rede de co-citação, os autores, números de citações e co-citações são: Barney (1991) com 47 citações e co-citado com 14 autores. Brandenburger e Nalebuf (1996) com 68 citações, e 16 co-citações. Bengtsson e Kock (2000) com 41 citações e 14 co-citações. Camerer (2003) com 4 citações e 3 co-citações. Dyer e Singh (1998) com 87 citações e 15 co-citações. Cohen e Levinthal (1990) com 49 citações e 12 co-citações. Eisenhardt (1989) com 87 citações e 15 co-citações. Fornell e Larcker (1981) com 34 citações e co-citado com 14 autores. Granovetter (1985) com 58 citações e 15 co-citações. Hamel e Prahalad (1989) com 49 citações e 13 co-citações. Harsaniy (1967) com 46 citações e 3 co-citações. Kogut e Zander (1992) com 41 citações e 14 co-citações. Pfeffer e Salancik (1978) com 34 citações e 13 co-citações. Porter (1980) com 28 citações e 14 co-citações. Shapley (1953) com 4 citações e 3 co-citações. Uzzi (1997) com 50 citações e 15 co-citações. Neumman e Morgenstern (1944) com 9 citações e 5 co-citações. Wasserman e 
Faust (1994) com 13 citações e 6 co-citações. Williamson (1985) com 59 citações e 16 co-citações. E por fim, Williamson (1975) com 44 citações e sendo co-citado com 15 autores na rede.

Figura 3 - Co-Citação de autores em Manufacturing Management

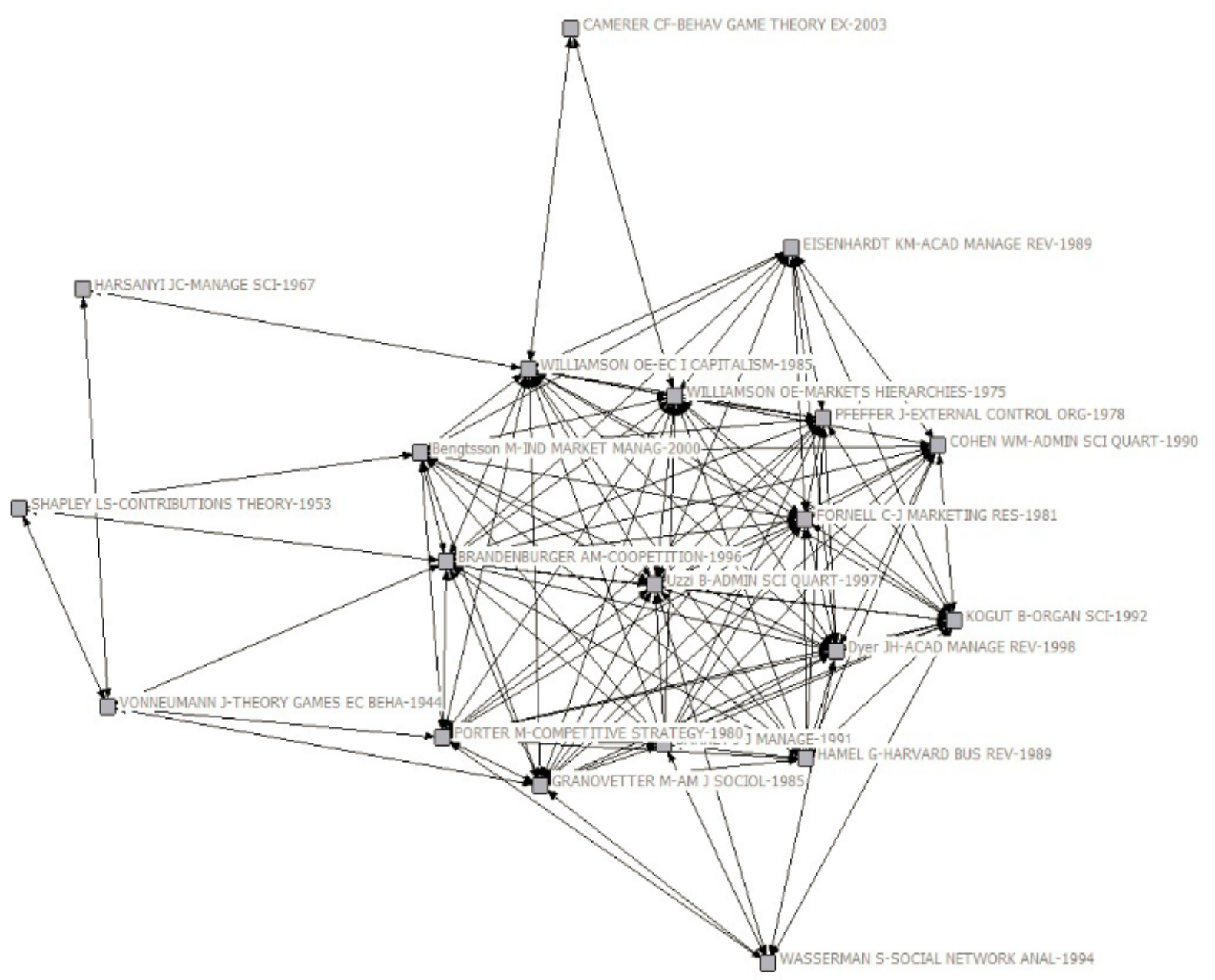

Fonte: Dados da Pesquisa, gerado através do UCINET ${ }^{\circledR}$.

Para Manufacturing Management as co-citações predominam com temas relacionados à dependência de recursos (PFEFFER; SALANCIK, 1978; WILLIAMSON, 1975; WILLIAMSON, 1985), questões voltadas para a competitividade no mercado (NEUMMAN; MORGENSTERN, 1944; BARNEY, 1991; HAMEL; PRAHALAD, 1989; PORTER, 1980; SHAPLEY, 1953; CAMERER, 2003). 
Autores ligados à cooperação empresarial e redes de empresas (HARSANIY, 1967; DYER; SINGH, 1998; UZZI, 1997; GRANOVETTER, 1985; KOGUT; ZANDER, 1992; WASSERMAN; FAUST, 1994). Destaca-se também a ideia de co-opetição (cooperação e competição ao mesmo tempo) como um tema emergente sendo abordado no contexto da gestão de manufatura (BRANDERBURGER; NALEBUFF, 1996; BENGTSSON; KOCK, 2000). Temas como a inovação (COHEN; LEVINTHAL, 1990), teoria da agência (EISENHARDT, 1989), modelos estruturais em variáveis não observáveis (FORNELL; LARCKER, 1981), fazem-se presentes na rede de cocitações.

\section{CONSIDERAÇÔES FINAIS}

De acordo com o objetivo da pesquisa de "analisar a produção acadêmica sobre o tema Gestão de Operações, em artigos publicados em periódicos internacionais indexados no ISI Web of Knowledge", pode-se afirmar que o mesmo foi atingido, devido o fato de que no decorrer do artigo foram apresentados os resultados e discussões de elementos como: periódicos e países onde foram publicados os artigos, a quantidade de artigos por ano de publicação, a abordagem dos autores mais co-citados e suas obras mais citadas, e os autores que mais publicaram artigos sobre o tema e suas obras.

Já sobre as perguntas problema dessa pesquisa "quais são as referências mais utilizadas nos estudos com o tema Gestão de Operações? E se existe alguma predominância nas referências bibliográficas utilizadas nos artigos sobre Gestão de Operações?", entende-se que foram respondidas. Sobre a segunda questão de pesquisa, pode-se dizer que não existe uma predominâncias nas referências utilizadas para os termos de pesquisa, e que não pode ser generalizado, pelo a amostragem de pesquisa ser limitada.

No tocante as preposições de pesquisa: (a) há concentração de publicações sobre Gestão de Operações em poucos periódicos, não pode ser confirmada devido à identificação de $41,57 \%$ do total de artigos analisados pertencem a periódicos que aparecem apenas uma vez em cada uma das pesquisas realizadas. Em contra partida uma representatividade de $30,48 \%$ do total dos artigos pertencem os 
periódicos: International Journal of Operations \& Production Management (50), International Journal of Production Research (36), International Journal of Production Economics (25), e o Journal of Operations Management (21), pelo alto grau de impacto que esses periódicos possuem e por sua tradição nessa área do conhecimento.

A preposição (b) existe predominância de publicações em um único país, foi confirmada devido à nítida concentração de publicações nos Estados Unidos, em todos os três termos de pesquisa utilizados. Ressaltando-se aqui a crescente quantidade de publicações de países asiáticos como a China, Japão e Taiwan. Já apreposição (c) há claramente um autor com maior número de publicações na área, foi refutada, pois não se identificou um autor que acumulou muitas publicações no período investigado, sendo no máximo identificado 3 (três) publicações para dois autores - Voss, C. e Gorman M. F. - no termo Operations Management. Para Production Management identificou-se Leung, S., C., H., com 5 (cinco) publicações. Os autores Godinho Filho, M. (2009 e 2011) e Sheu. D., D. (2003 e 2004) com 2 (duas) publicações cada para o termo Manufacturing Management.

E por fim, sobre a preposição (d) as publicações na área cresceram conforme o passar dos anos, não pôde ser confirmada, devido os resultados apontarem oscilações para mais e para menos a cada ano investigado, com aumentos de um ano para o outro, seguidos de queda nas publicações. Salienta-se que o ano de 2012 apresentou somente 12 (doze) publicações, devido à pesquisa ter sido realizada em abril de 2012, e o ano com maior número de publicações foi o de 2007 com 60 publicações no total.

Sobre os autores mais citados e co-citados nas referências bibliográficas, destacam-se a influência de Williamson (1975) que associa a economia de mercado as teorias organizacionais na forma de um institucionalismo econômico. Assim as transações entre empresas são conduzidas através do mercado e das hierarquias e isso dependerá do potencial de impacto da informação. Essa situação se evidencia quando as verdadeiras condições subjacentes das transações são de conhecimento por uma ou mais partes envolvidas na transação. Para Dyer e Singh (1998) economia dos custos de transação está associada à forma e intensidade de cooperação entre as empresas, bem como com a relação de fatores econômicos e 
tecnológicos e com as relações sociais, que moldam esse comportamento (GRANOVETTER, 1985; UZZI, 1997).

A pesquisa limita-se pelo fato de não abranger todos os artigos publicados disponíveis nas bases de dados, o que sugere a possibilidade de análises complementares. Nesse sentido, tanto com a formação de um comparativo, como a ampliação a amostra poderia proporcionar generalizações mais precisas sobre o tema geral e seus correlatos aqui estudados.

\section{REFERÊNCIAS}

ARAÚJO, C.A. Bibliometria: evolução histórica e questões atuais. Em Questão, Porto Alegre, v. 12, n. 1, p. 11-32, jan./jun. 2006.

ARORA, A.; GAMBARDELLA, A. Complementarity and external linkages: the strategies of the large firms in biotechnology, Journal of Industrial Economics, 38, p. 361-379, 1990.

AXEROLD, R. The Evolution of Cooperation. New York: Basic Books, 1984.

BARNEY, J. Firm Resources and Sustained Competitive Advantage. Journal of Management, v. 17, p. 99-120, 1991.

BENGTSSON, M.; KOCK, S. Coopetition in Business Networks - to Cooperate and Compete Simultaneously. Industrial Marketing Management, v. 29, p. 411-426, 2000. http://dx.doi.org/10.1016/S0019-8501(99)00067-X

BIVIN, D., G. Firm performance under just-in-time and traditional proxies for profit maximization. International Journal of Production Economics, v. 81-81, n. 11, p. 141152, 2003.

BIVIN, D., G. Has production management improved since 1984 ? Economic Inquiry, v. 44, n. 4, p. 671-688, 2006. http://dx.doi.org/10.1093/ei/cbj038

BIVIN, D., G. Industry evidence of enhanced production stability since 1984 . International Journal of Production Economics, v. 103, n. 1, p. 438-448, 2006.

http://dx.doi.org/10.1016/i.ijpe.2005.09.007

BIVIN, D., G. Production stability in a supply-chain environment. International Journal of Production Economics, v. 114, n. 1, p. 265-275, 2008. http://dx.doi.org/

10.1016/j.ijpe.2008.02.007

BORBA, M. L. de; HOELTGEBAUM, M.; SILVEIRA, A. A produção científica em Empreendedorismo: análise do academy of management meeting: 1954-2005. RAM Revista de Administração Mackenzie, São Paulo-SP, v. 12, n. 2, p. 169-206, Mar./Abr., 2011. http://dx.doi.org/10.1590/S1678-69712011000200008 
BRANDENBURGER, A., M.; NALEBUFF, B., J. Co-opetition: A revolutionary mindset that combines competition and cooperation - The Game Theory strategy that' changing the game of business. Curreney Book, Doubleday, New York, 1996.

CAMERER C., F. Behavioral Game Theory. Russell Sage Foundation, New York, Princeton University Press, 2003.

CLARK, K.; FUJIMOTO, T. Product Development Performance. Boston: Harvard Business School Press, 1991.

COHEN W. M.; LEVINTHAL, D., A. Absorptive capacity: A new perspective on learning and innovation. Admin. Sci. Quart. v. 35, p.128-152, 1990.

COLLINS, J.; HUSSEY, R. Pesquisa em Administração. Porto Alegre: Bookman, 2005. DIENSTMANN, J. S.; LACERDA, R. T. de O.; ENSSLIN, L.; ENSSLIN, S. R. Gestão da inovação e avaliação de desempenho: processo estruturado de revisão da literatura. Revista Produção Online, v. 14, n. 1, p. 2-30, 2014. http://dx.doi.org/10.14488/16761901.v14.i1.1042

DOZ, Y. L. The Evolution of Cooperation in Strategic Alliances: Initial Conditions or Learning Processes? Strategic Management Journal, v. 17, p. 55-83, 1996.

http://dx.doi.org/10.1002/smj.4250171006

DYER, J. H.; SINGH, H. The relational view: Cooperative strategy and sources of interorganizational competitive advantage. Academy of Management Review, v. 23, v. 4, p. 660-679, 1998.

ENDERSON, R., M.; CLARK, K., B. Architectural Innovation: the reconfiguration of existing product technologies and the failure of establishment firms. Administrative Science Quarterly, v.35, n.1, p. 9-30, Mar. 1990.

EISENHARDT, K. Agency theory: an assessment and review. Academy of Management Review, v.14, p.57-74, 1989.

FEHR, E.; SCHMIDT, K., M. A theory of fairness, competition, and cooperation. The Quarterly Journal of Economics, v. 114, n. 3, p. 817-868, 1999.

FERDOWS, K.; MEYER, A. de. Lasting Improvements in Manufacturing Performance: in Search of a New Theory. Journal of Operation Management, v. 9, n. 2, 1990.

FORNELL, C.; LARCKER, D., F. Structural Equation Models with Unobservable Variables and Measurement Error - Algebra and Statistics. Journal of Marketing Research, v. 18, n. 3, p. 382-388, 1981.

FRANCISCHINI, G. P. Administração de materiais e do patrimônio. São Paulo: Pioneira Thomson, 2004.

FUSCO, J. P. A; SACOMANO, J. B. Operações e Gestão Estratégica da Produção. São Paulo, Arte \& Ciência, 2007. 
GABRIELE, P. D.; TREINTA, F. T.; FARIAS FILHO, J. R. de; SOUZA, M. C. de; TSCGAFFON, P. B.; BRANTES, S. R. Sustentabilidade e vantagem competitiva estratégica: um estudo exploratório e bibliométrico. Revista Produção Online, v. 12, n. 3, p. 729-755, 2012. http://dx.doi.org/10.14488/1676-1901.v12i3.975

GODINHO FILHO, M.; FERNANDES, F., C., F. Strategic Paradigms for Manufacturing Management (Spmm): Key Elements and Conceptual Model. International of industrial Engineering - Theory Application and Practice, v. 16, n. 2, p. 147-159, 2009.

GODINHO FILHO, M.; UZSOY, R. The effect of shop floor continuous improvement programs on the lot size-cycle time relationship in a multi-product single-machine environment. International Journal of Advanced Manufacturing Technology, v. 52, n. 5, p. 669-681, 2011. http://dx.doi.org/10.1007/s00170-010-2770-8

GORMAN, M., F. OM Forum: Evaluating operations management-related journals via the author affliation index - Do professors at top US business schools do what they say?

Manufacturing \& Service Operations Management, v. 9, n.1, p. 51-53, 2007.

GORMAN, M., F. Student Reactions to the Field Consulting Capstone course in Operations Management at the University of Dayton. Interfaces, v. 41, n. 6, p. 564-577, nov-dez, 2011. http://dx.doi.org/10.1287/inte.1110.0567

GORMAN, M., F. The University of Dayton Operations Management Capstone Course: Undergraduate Student Field Consulting Applies Theory to Practica. Interfaces, v. 40, n. 6, p. 432-443, nov-dez, 2011. http://dx.doi.org/10.1287/inte.1110.0567

GRANOVETTER, M. Economic Action and Social Structure: The Problem of Embeddedness. The American Journal of Sociology, vol. 91, n. 3, p. 481-510, 1985.

GULATI, R. Social structure and alliance formation pattern: A longitudinal analysis. Administrative Science Quarterly, v. 40, p. 619-642, 1995.

HAGEDOOM, J. Understanding the Rationale of Strategic Technology Partnering since the Early Seventies. Review of Industrial organization, v. 11, p. 601-616, 1993.

HAMEL, G. Competition for Competence and Inter-Partner Learning within International Strategic Alliances. Strategic Management Journal, v. 12, p. 83-103, 1991. http://dx.doi.org/10.1002/smj.4250120908

HAMEL, G.; PRAHALAD, C. K. Strategic Intent. Harvard Business Review, p.17-30, 1989. HARSANYI, J. Games with incomplete information played bayesian players. Management Science, v. 14, p. 159-182, 1967. http://dx.doi.org/10.1287/mnsc.14.5.320

HALL, R. W. Excelência na Manufatura. São Paulo, IMAM, 1988.

HILL, T. Manufacturing Strategy - the strategic management of the manufacturing function. London: Macmillan Press Ltd., 1993.

HUANG, C., J.; SHEU, D., D. Fuzzy multi-attribute analysis for evaluation of manufacturing management performance of notebook computer plants - A case study. International 
Journal of Industrial Engineering - Theory Applications and Practice, v. 11, n. 2, p. 178-186, 2004.

KAMIEN, M., I.; SCHWARTZ, N., L. Market structure and innovation. Cambridge: Cambridge University Press, 1982.

KARLSSON, C.; VOSS, C. Operations management associations in Europe: a history. International Journal of Operations \& Production Management, v. 29, n. 11, p. 10931108, 2009. http://dx.doi.org/10.1108/01443570911000140

KNOFF, L. C.; LACERDA, R. T. de O.; ENSSLIN, L.; ENSSLIN, S. R. Mapeamento de publicações científicas sobre estratégia de manufatura: uma abordagem baseada em processos. Revista Produção Online, v. 14, n. 1, p. 403-429, 2014.

http://dx.doi.org/10.14488/1676-1901.v14i2.1043

KOGUT, B.; ZANDER, U. Knowledge of the firm, combinative capabilities, and the replication of technology. Organization Science, v. 3, n. 3, p. 383-397, 1992.

http://dx.doi.org/10.1287/orsc.3.3.383

LAWRENCE, P., R.; LORSCH, J., W. Organization and Environment: Managing Differentiation and Integration. Boston, MA: Harvard University, 1967.

LEUNG, S., C., H.; WU, Y.; LAI, K., K. A stochastic programming approach for multi-site aggregate production planning. Journal of the Operational Research Society, v. 57, n. 2, p. 123-132, 2006. http://dx.doi.org/10.1057/palgrave.jors.2601988

LEUNG, S., C., H; NG, W., L. A stochastic programming model for production planning of perishable products with postponement. Production Planning \& Control, v. 18, n. 3, p. 190202, 2007. http://dx.doi.org/10.1080/09537280601009047

LEUNG, S., C., H; NG, W., L. A goal programming model for production planning of perishable products with postponement. Computers \& Industrial Engineering, v. 53, n. 3, p. 531-541, 2007.http://dx.doi.org/doi:10.1016/i.cie.2007.05.010

LEUNG, S., C., H; TSANG, O., S., S.; NG, W., L. A robust optimization model for multi-site production planning problem in na uncertain environment. European Journal of Operational Research, v. 181, n.1, p. 224-238, 2007. http://dx.doi.org/10.1016/i.ejor.2006.06.011

LEUNG, S., C., H.; LAI, K., K.; NG, W., L. A robust optimization model for production planning of perishable products. Journal of the Operational Research Society, v. 58, n. 4, p. 413-422, 2007. http://dx.doi.org/10.1057/palgrave.jors.2602159

LOPES, A. P.; CARVALHO, M. M. de; FLEURY, A. C. C. Redes Sociais e cooperação: um estudo bibliométrico. Revista Produção Online, v. 13, n. 2, p. 634-654, 2013. http://dx.doi.org/10.14488/1676-1901.v13i2.1231

MARTINS, G., S.; ROSSONI, L.; CSILLAG, J., M.; MARTINS, M., E.; PEREIRA, S., C., F. Gestão de Operações no Brasil: uma análise do campo científico a partir da rede social de pesquisadores. RAE - Revista de Administração de Empresas Eletrônica, v. 9, n.2, art. 8, jul/dez, 2010. http://dx.doi.org/10.1590/S1676-56482010000200004 
MEREDITH, J., R.; STEWARD, M., D.; LEWIS, B., R. Knowledge dissemination in operations management: Published perceptions versus academic reality. International Journal of Management Science, v. 39, n. 4, p. 435-446, 2011.

MOREIRA, D. A. Administração da Produção e Operações. São Paulo, Cengage Learning, 2008.

MORGAN, R., M.; HUNT, S. The Commitment-Trust Theory of Relationship Marketing. Journal of Marketing, v. 58, 1994.

NEUMANN, J. von; MORGENSTERN, O. Theory of Games and Economic Behavior. Princeton University, 1944.

NORTH, D. Institutions: Institutional Change and Economic Performance. Cambridge: Cambridge University Press, 1990.

PARKHE, A. Strategic alliance structuring: A game theoretic and transaction cost examination of interfirm cooperation. Academy of Management Journal, v. 36, n. 4, p. 794829, 1993. http://dx.doi.org/10.2307/256759

PAULISTA, P.; CAMPOS, D.; TURRIONI, J. Análise Bibliométrica da Gestão do Conhecimento. In Anais: ENEGEP, São Carlos, 2010.

PFEFFER, J.; SALANCIK, G., R. The External Control of Organizations: A Resource Dependence Perspective. New York, NY, Harper and Row, 1978.

PINTO, M., B.; PINTO, J., K.; PRESCOTT, J., E. Antecedents and consequences of project team cross-functional cooperation. Management Science, v. 39, p. 1281-1297, 1993. http://dx.doi.org/10.1287/mnsc.39.10.1281

PIRES, S., R., I. Integração do planejamento e controle da produção a uma estratégia de manufatura. Tese de Doutorado - Escola de Engenharia de São Carlos, Universidade de São Paulo, São Carlos, 1994.

PIRES, S., R., I. Gestão da Cadeia de Suprimentos - Supply Chain Management Conceitos, Estratégias, Práticas e Casos. 2. ed., São Paulo: Editora Atlas, 2009. PORTER, M. Competitive Strategy. Free Press, 1980.

RABIN, M. Incorporating fariness Into Game Theory and Economics. University of California-Berkeley, 1992.

ROESCH, S. M. Projetos de Estágio e de Pesquisa em Administração. São Paulo: Atas, 1999.

RUNGTUSANATHAM, M., J.; CHOI, T., Y.; HOLLINGWORTH, D., G. Survey research in operations management: historical analyses. Journal of Operations Management, v. 21, n. 4, p. 475-488, 2003. http://dx.doi.org/10.1016/S0272-6963(03)00020-2

SCHELLING, T., C. The Strategy of Conflict. Harvard U Press, 1960. 
SHAPLEY, L., S. The value of an N-Person Game: In Contributions to the Theory of Games. Princeton University Press, 1953.

SHEU, D., D.; PENG, S., L. Assessing manufacturing management performance for notebook computer plants in Taiwan. International Journal of Production Economics, v. 84, n. 2, p. 215-228, 2003. http://dx.doi.org/10.1016/S0925-5273(02)00428-0

SKINNER, W. Manufacturing - missing link in corporate strategy. Harvard Business Review, HBR Publishing, Boston, 136-145, 1969.

SLACK, N.; LEWIS, M. Operations Strategy. Prentice Hall, London, 2002.

SLACK, N. et al. Administração da Produção. 3. ed., São Paulo: Editora Atlas, 2009.

TEECE, D.; RUMELT, R.; SCHENDEL, D. (eds.). Fundamental Issues in Strategy. Havard Business School Press, Boston, MA, 1992.

TEECE, D., J. Profiting from technological innovation: Implications for integration, collaboration, licensing, and public policy. Research Policy, 1986.

http://dx.doi.org/10.1016/0048-7333(86)90027-2

TENÓRIO, F. A. G.; SILVA, D. E. P. da; DACORSO, A. L. R. Inovação e tomada de decisão no processo de logística reversa: uma análise bibliométrica. Revista Produção Online, v. 14, n. 2, p. 593-616, 2014. http://dx.doi.org/10.14488/1676-1901.v14i2.1434

THOMPSON, J., D. Organizations in Action. McGraw Hill: New York, 1967.

UZZI, B. Social structure and competition in interfirm networks: the paradox of embeddedness. Administrative Science Quarterly, v. 4, p. 35-67, 1997.

http://dx.doi.org/10.2307/2393808

VOSS, C., A. Learning from the first Operations Management textbook. Journal of Operations Management, v. 25, p. 239-247, 2007.

http://dx.doi.org/10.1016/i.jom.2006.05.013

VOSS, C.; TSIKRIKTSIS, N.; FROHLICH, M. Case research in operations management. International Journal of Operations \& Production Management, v. 22, n. 2 p. 195-219, 2002. http://dx.doi.org/10.1108/01443570210414329

WASSERMAN, S.; FAUST, K. Social Network Analysis: Methods and Applications. Cambridge, Massachusetts: Cambridge University Press, 1994.

WILLIAMSON, O., E. The Economic Institutions of Capitalism. New York: The Free Press, 1985.

WILLIAMSON, O., E. Market and Hierarchies: Analysis and Antitrust Implications. New York: The Free Press, 1975.

WHEELWRIGHT, S.C.; HAYES, R.H. Competing through manufacturing. Harvard Business Review, New York, v.63, n.1, p. 99-109, 1985. 
WU, Y.; FRIZELLE, G.; EFSTATHIOU J. A study on the cost of operational complexity in customer-supplier systems. International Journal of Production Economics, v. 106, n. 1, p. 217-229, 2007. http://dx.doi.org/10.1016/i.ijpe.2006.06.004

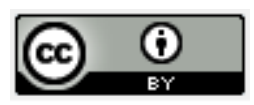

Artigo recebido em 22/04/2013 e aceito para publicação em 19/12/2014

DOI: http://dx.doi.org/ 10.14488/1676-1901.v15i1.1574 